\title{
Cross soliton and breather soliton for the $(3+1)$-dimensional Yu-Toda-Sasa-Fukuyama equation
}

\author{
Zhiqiang $\mathrm{Pu}^{1 *}$ (D) and Zhigang Pan ${ }^{2 *}$
}

\section{"Correspondence: pzq@mtc.edu.cn; panzhigang@swjtu.edu.cn ${ }^{1}$ School of Information Engineering, Mianyang Teachers' College, Mianyang, China \\ ${ }^{2}$ School of Mathematics, Southwest Jiaotong University, Chendu, P.R. China}

\begin{abstract}
Cross-soliton solution, breather soliton, periodic solitary solution, and doubly periodic solution are obtained by using an extended homoclinic test approach with perturbation parameter $u_{0}$ and complexity of parameters, respectively. Dynamical feature of cross soliton flow including degeneracy of soliton with different directions, retroflexion of breather soliton for YTSF equation is investigated using the parameter perturbation method. Result shows that the value range of constant equilibrium solution can determine the dynamics of cross soliton for a higher dimensional nonlinear system.
\end{abstract}

MSC: $35 C 08 ; 74 J 35$

Keywords: YTSF equation; Degeneracy; Cross soliton; Retroflexion

\section{Introduction}

It is well established now that the higher-dimensional nonlinear wave fields have richer behavior than one-dimensional ones. It was verified that the existence of two solitons having the structures peculiar to a higher-dimensionality may contribute to the variety of the dynamics of nonlinear waves [1-3]. Thereby, seeking for exact solution and studying dynamical behavior [4-7] of solutions are very significant in physics, mathematics, and nonlinear science fields for understanding the complexity and variety of dynamics determined by high-dimensional nonlinear evolution equation [8-10]. In soliton theory, the soliton solutions are obtained by the use of the inverse scattering method, Bäcklund transformation, Darboux transformation, Painlevè method, Hirota method, the tanh method, the generalized Riccati equation expansion method, homoclinic test method, etc. [11-18]. In this work, we would like to use the parameter perturbation method for seeking dynamical feature of soliton solution for the $(3+1)$-dimensional Yu-Toda-Sasa-Fukuyama (YTSF) equation.

The YTSF equation has been presented as

$$
\left(-4 u_{t}+\Phi(u) u_{z}\right)_{x}+3 u_{y y}=0, \quad \Phi(u)=\partial_{x}^{2}+4 u+2 u_{x} \partial_{x}^{-1}
$$

(c) The Author(s) 2019. This article is distributed under the terms of the Creative Commons Attribution 4.0 International License (http://creativecommons.org/licenses/by/4.0/), which permits unrestricted use, distribution, and reproduction in any medium, provided you give appropriate credit to the original author(s) and the source, provide a link to the Creative Commons license, and indicate if changes were made. 
where $\partial_{x}^{-1}$ represents the integral with respect to $x$. This equation was introduced by $\mathrm{Yu}$, Toda, Sasa, and Fukuyama [19] as a generalization from the Bogoyavlenskii-Schif equation [20]. Linearly traveling solitary wave solution for Eq. (1) was found by using tanhfunction method [21]. The optimistic quadratic polynomial function lump solutions, some soliton-like solutions, nontravelling wave solutions, and a new kink solution for the potential form of Eq. (1) were obtained by a Backlund transformation, an auto-Backlund transformation, and the extended homoclinic test method [22-25].

This paper focuses on the exact solutions and spatiotemporal dynamics of solution for Eq. (1). Three types of exact solutions including cross-soliton, breather soliton (periodic solitary solution), and doubly periodic solutions to YTSF equation are constructed by bilinear form and extended homoclinic test approach, a technique of searching for exact homoclinic orbit solution, for nonlinear integrable equation [23, 26]. It is explicitly exhibited that the dynamical feature of the solutions is different on the both sides of an arbitrary constant equilibrium solution (point) of the YTSF equation. Cross-soliton solution is degenerated into periodic solitary wave and breather soliton with different directions and even double periodic solution when the equilibrium point $u_{0}$ varies from one side of $-\frac{1}{6}\left(4 \alpha+c p^{2}\right)$ or $-\frac{2 \alpha-2 c p^{2}}{3 c}$ to another side, where $\alpha$ is the propagation velocity of wave, $p$ is a wave number, and $c$ is a fixed constant. To the best of our knowledge, these results have not been studied yet.

\section{Cross soliton of YTSF}

Using the potential transformation $u=v_{x}$, a $(3+1)$-dimensional potential YTSF equation has been derived [27, 28]:

$$
-4 v_{x t}+v_{x x x z}+4 v_{x} v_{x z}+2 v_{x x} v_{z}+3 v_{y y}=0
$$

We suppose that $\eta=x+c z-\alpha t$, then Eq. (2) can be transformed into

$$
4 \alpha v_{\eta \eta}+c v_{\eta \eta \eta \eta}+3 c\left(v_{\eta}^{2}\right)_{\eta}+3 v_{y y}=0
$$

By using Painlevé analysis Eq. (3), we suppose

$$
\left\{\begin{array}{l}
v=u_{0}+2(\ln f)_{\eta}, \\
f=b_{1} e^{-p_{1}\left(\beta_{1} y+\mu_{1} \eta\right)}+b_{0} \cos \left(p_{2}\left(\beta_{2} y+\mu_{2} \eta\right)\right)+b_{2} e^{p_{1}\left(\beta_{1} y+\mu_{1} \eta\right)}
\end{array}\right.
$$

where all of $p_{1}, p_{2}, \beta_{1}, \beta_{2}, \mu_{1}, \mu_{2}, b_{0}, b_{1}, b_{2}, k_{1}, k_{2}$, and $k_{3}$ are parameters to be determined later. Substituting Eq. (4) into Eq. (3) and equating all the coefficients of different powers of $\cos \left(p_{2}\left(\beta_{2} y+\mu_{2} \eta\right)\right), \sin \left(p_{2}\left(\beta_{2} y+\mu_{2} \eta\right)\right), e^{j p_{1}\left(\beta_{1} y+\mu_{1} \eta\right)}, j=1,2,3,4$ and constant term to zero, 
we can obtain a set of algebraic equations:

$$
\left\{\begin{array}{l}
2 c \mu_{1}^{5} p_{1}^{4}-2\left(10 c p_{2}^{2} \mu_{1}^{2} \mu_{2}^{2}-2\left(3 c k_{2}+2 \alpha\right) \mu_{1}^{2}-3 \beta_{1}^{2}\right) \mu_{1} p_{1}^{2} \\
\left.\quad+2\left(5 c p_{2}^{2} \mu_{1} \mu_{2}^{4}-6\left(3 c k_{2}+2 \alpha\right) \mu_{2}^{2}-3 \beta_{2}^{2}\right) \mu_{1}-6 \mu_{2} \beta_{1} \beta_{2}\right) p_{2}^{2}=0, \\
c \mu_{2}^{5} p_{2}^{4}-2\left(5 c p_{1}^{2} \mu_{1}^{2}+3 c k_{2}+2 \alpha\right) \mu_{2}^{3} p_{2}^{2}+6 \mu_{1} \beta_{1} \beta_{2} p_{1}^{2} \\
\quad+\left(5 c p_{1}^{4} \mu_{1}^{4}+\left(\left(18 c k_{2}+12 \alpha\right) \mu_{1}^{2}+3 \beta_{1}^{2}\right) p_{1}^{2}-3 p_{2}^{2} \beta_{2}^{2}\right) \mu_{2}=0, \\
\left.\left(b_{0}^{2}-\frac{1}{4} b_{1} b_{2}\right) c p_{2}^{4} \beta_{2}^{5}-\left(\frac{3}{2}\left(u_{1}^{2} p_{1}^{2}-k_{2}\right) c+\alpha\right) b_{1} b_{2}+b_{0}^{2}\left(\frac{3}{2} c k_{2}+\alpha\right)\right) p_{2}^{2} \mu_{2}^{3} \\
\quad+\frac{9}{2} b_{1} b_{2} \beta_{1} \beta_{2} \mu_{1} p_{1}^{2}+\frac{3}{4}\left(3 b_{1} b_{2} \mu_{1}^{2} p_{1}^{2}\left(3 c \mu_{1}^{2} p_{1}^{2}+6 c k_{2}+4 \alpha\right)\right. \\
\left.\quad+b_{1} b_{2}\left(3 \beta_{1}^{2} p_{1}^{2}+\beta_{2}^{2} p_{2}^{2}\right)-b_{0}^{2} \beta_{2}^{2} p_{2}^{2}\right) \mu_{2}=0, \\
2 c b_{1} b_{2} \mu_{1}^{5} p_{1}^{4}+\frac{1}{2}\left(-c b_{0}^{2} \mu_{2}^{2} p_{2}^{2}+2 b_{1} b_{2}\left(3 c k_{2}+2 \alpha\right)\right) p_{1}^{2} \mu_{1}^{3} \\
\quad-\frac{3}{4} \mu_{2} \beta_{1} \beta_{2} b_{0}^{2} p_{2}^{2} \frac{1}{8}\left(2 c b_{0}^{2} \mu_{2}^{2} p_{2}^{2}\left(4 c \mu_{2}^{2} p_{2}^{2}-9 c k_{2}-6 \alpha\right)\right. \\
\left.\quad-3 b_{0}^{2} \beta_{2}^{2} p_{2}^{2}+12 b_{1} b_{1} \beta_{1}^{2} p_{1}^{2}\right) \mu_{1}=0 .
\end{array}\right.
$$

Solving the set of algebraic equations for $p, \Omega, b_{1}, b_{2}, A$ yields the exact solution of Eq. (1) as follows:

$$
u=u_{0}+\frac{2 c p^{2}\left[b_{1}^{2}+b_{1}\left(e^{i p \eta}+e^{-i p \eta}\right)\left(b_{2} e^{\Omega y+\gamma}+e^{-\Omega y-\gamma}\right)\right]}{\left[b_{1}\left(e^{i p \eta}+e^{-i p \eta}\right)+\left(b_{2} e^{\Omega y+\gamma}+e^{-\Omega y-\gamma}\right)\right]^{2}},
$$

where parameters $A, p, \Omega, \gamma, b_{1}$, and $b_{2}$ satisfy the dispersive relations

$$
A=0, \quad 3 \Omega^{2}=-c p^{4}-\left(4 \alpha+6 u_{0}\right) p^{2}, \quad b_{1}^{2}=\frac{4 \Omega^{2} b_{2}}{\Omega^{2}-c p^{4}} .
$$

It is obvious that $u_{0}<-\frac{1}{6}\left(c p^{2}+4 \alpha\right)$ is required so that the conditions $\Omega^{2}>0, b_{1}^{2}>0$, and $0<p^{2}<-\frac{4 \alpha+6 u_{0}}{c}$ can be satisfied in Eq. (9). Notice that $u_{0}$ can be taken as an arbitrary real number because the speed of propagating wave $\alpha$ can be arbitrary (only corresponding to the direction and speed propagating wave on the $x$-axis).

Taking $\eta=i(x+c z-\alpha t)$ into Eq. (6), the exact solution to the YTSF equation is expressed by

$$
u(x, y, z, t)=\frac{2 c p^{2}\left(b_{1}^{2}+H_{1} H_{2}\right)}{\left(H_{1}+H_{2}\right)^{2}},
$$

where

$$
\left\{\begin{array}{l}
u_{0}<\operatorname{Min}\left\{-\frac{1}{6}\left(4 \alpha+c p^{2}\right),-\frac{1}{3}\left(2 \alpha+2 c p^{2}\right)\right\} \\
0<p^{2}<-\frac{4 \alpha+6 u_{0}}{c} \\
3 \Omega^{2}=-c p^{4}-\left(4 \alpha+6 u_{0}\right) p^{2} \\
b_{1}^{2}==\frac{4 \Omega^{2} b_{2}}{\Omega^{2}-c p^{4}} \\
H_{1}=b_{1}\left(e^{p(x+c z-\alpha t)}+e^{-p(x+c z-\alpha t)}\right) \\
H_{2}=b_{2} e^{\Omega y+\gamma}+e^{-\Omega y-\gamma}
\end{array}\right.
$$


Figure 1 The cross-soliton solution (10) with $u_{0}=-0.5, c=-1, \alpha=\frac{1}{4}, p=0.6, t=-1, z=0.1$

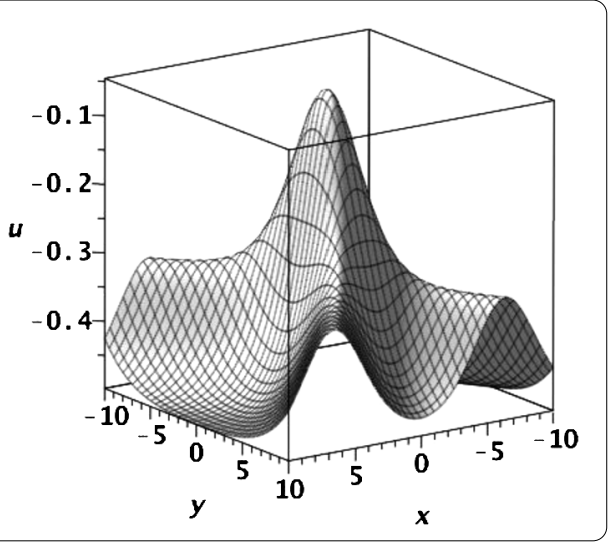

Especially, taking $\gamma=0, b_{2}=1$ in expression Eq. (8) of $u$, the cross-soliton solution to YTSF is obtained as follows:

$$
u(x, y, z, t)=\frac{c p^{2}\left[b_{1}^{2}+4 b_{1} \cosh (p(x+c z-\alpha t)) \cosh (\Omega y)\right]}{2\left[b_{1} \cosh (p(x+c z-\alpha t))+\cosh (\Omega y)\right]^{2}} .
$$

The solution represented by Eq. (10) is a cross soliton which contains one soliton and one solitary wave with different propagation direction (see Fig. 1).

\section{Periodic soliton of YTSF}

Let $\Omega=i \Omega_{1}$ in Eq. (7), where $\Omega_{1}$ is a real number, then replacing $\Omega_{1}$ with $\Omega$, Eq. (7) changes into

$$
A=0, \quad 3 \Omega^{2}=c p^{4}+\left(4 \alpha+6 u_{0}\right) p^{2}, \quad b_{1}^{2}=\frac{4 \Omega^{2} b_{2}}{\Omega^{2}+c p^{4}} .
$$

Here, it is obvious that $u_{0}>\operatorname{Max}\left\{-\frac{1}{6}\left(4 \alpha+c p^{2}\right),-\frac{1}{3}\left(2 \alpha+2 c p^{2}\right)\right\}$ is required so that the conditions $\Omega^{2}>0$ and $b_{1}^{2}>0$ can be satisfied. Notice that $u_{0}$ also can be taken as an arbitrary real number by the same argument as in the above cross-soliton case. Taking $\Omega=i \Omega_{1}$ in Eq. (10), replacing $\Omega_{1}$ with $\Omega$, we get the solution of Eq. (1) as follows:

$$
u(x, y, z, t)=\frac{c p^{2}\left[b_{1}^{2}+4 b_{1} \cosh (p(x+c z-\alpha t)) \cos (\Omega y)\right]}{2\left[b_{1} \cosh (p(x+c z-\alpha t))+\cos (\Omega y)\right]^{2}},
$$

where

$$
\left\{\begin{array}{l}
u_{0}>-\frac{1}{6}\left(4 \alpha+c p^{2}\right), \\
3 \Omega^{2}=c p^{4}+\left(4 \alpha+6 u_{0}\right) p^{2}, \\
b_{1}^{2}=\frac{4 \Omega^{2} b_{2}}{\Omega^{2}+c p^{4}} .
\end{array}\right.
$$

The solution represented by Eq. (12) is a periodic solitary solution which contains one solitary wave and one periodic wave, its amplitude occurs periodically, oscillation varying with variable $y$ (see Fig. 2).

It is interesting that $u_{0}$ plays an important role in the dynamics of cross soliton, cross soliton degenerates into periodic solitary solution when $u_{0}$ passes through $-\frac{1}{6}\left(4 \alpha+c p^{2}\right)$ 
Figure 2 The periodic solitary solution (12) with $u_{0}=\frac{-1}{18}, c=-1, \alpha=\frac{1}{2}, p=\sqrt{\frac{1}{3}}, t=-1, z=0$

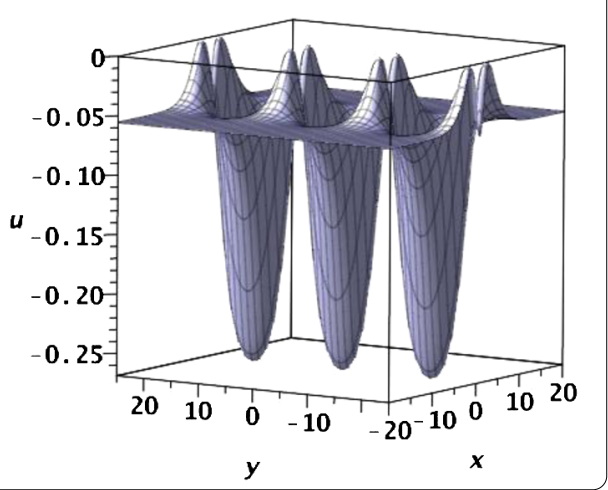

from the left side to the right side. This shows a kind of bifurcation phenomenon with parameter $u_{0}$ at the special value $-\frac{1}{6}\left(4 \alpha+c p^{2}\right)$.

\section{Breather soliton of YTSF}

Setting $p=i p_{1}$ in Eq. (7), where $p_{1}$ is a real number, then replacing $p_{1}$ with $p$, Eq. (7) changes into

$$
A=0, \quad 3 \Omega^{2}=-c p^{4}+\left(4 \alpha+6 u_{0}\right) p^{2}, \quad b_{1}^{2}=\frac{4 \Omega^{2} b_{2}}{\Omega^{2}-c p^{4}} .
$$

In this case, $u_{0}>\frac{1}{6}\left(c p^{2}-4 \alpha\right)$ is required so that the conditions $\Omega>0$ and $b_{1}^{2}>0$ are satisfied. Taking $p=i p_{1}$ in Eq. (10) and replacing $p_{1}$ with $p$, we get the solution of Eq. (1) as follows:

$$
u(x, y, z, t)=\frac{c p^{2}\left[b_{1}^{2}+4 b_{1} \cos (p(x+c z-\alpha t)) \cosh (\Omega y)\right]}{2\left[b_{1} \cos (p(x+c z-\alpha t))+\cosh (\Omega y)\right]^{2}}
$$

where

$$
\left\{\begin{array}{l}
u_{0}>\frac{1}{6}\left(c p^{2}-4 \alpha\right), \\
3 \Omega^{2}=-c p^{4}+\left(4 \alpha+6 u_{0}\right) p^{2}, \\
b_{1}^{2}=\frac{4 \Omega^{2} b_{2}}{\Omega^{2}-c p^{4}} .
\end{array}\right.
$$

The solution represented by Eq. (15) is a breather soliton which is a soliton when the trajectory defined by Eq. (15) propagating along the straight line $x+c z-\alpha t=$ constant, and it also is a periodic wave as $y=$ constant (see Fig. 3).

Combining the above results, we show two important dynamical features of cross soliton, cross soliton degenerates into periodic solitary wave when $u_{0}$ passes through $\frac{1}{6}\left(c p^{2}-4 \alpha\right)$ from the left side to the right side.

\section{Doubly periodic solution}

Setting $\Omega=i \Omega_{1}, p=i p_{1}$ in Eq. (7) and then replacing $\Omega_{1}$ with $\Omega$, $p_{1}$ with $p$, Eq. (7) changes into

$$
A=0, \quad 3 \Omega^{2}=c p^{4}-\left(4 \alpha+6 u_{0}\right) p^{2}, \quad b_{1}^{2}=\frac{4 \Omega^{2} b_{2}}{\Omega^{2}+c p^{4}} .
$$


Figure 3 The periodic solitary solution (15) with $u_{0}=0, c=-1, \alpha=\frac{1}{4}, p=0.6, t=-1, z=1$

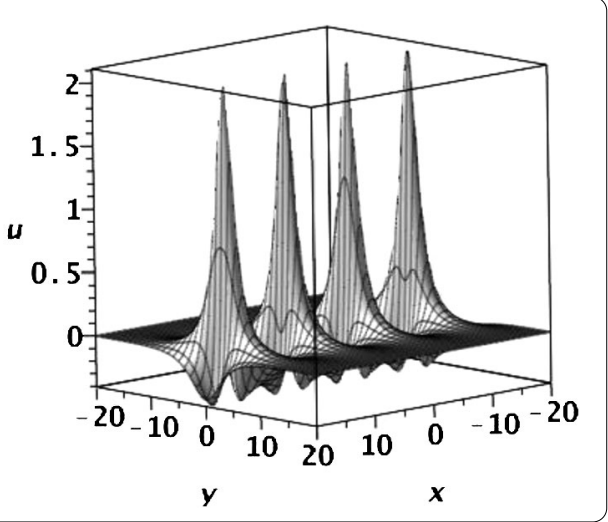

Here, $u_{0}<\frac{1}{6}\left(c p^{2}-4 \alpha\right)$ is required so that the conditions $\Omega>0$ and $b_{1}^{2}>0$ can be satisfied. Taking $\Omega=i \Omega_{1}, p=i p_{1}$ in Eq. (10) and replacing $p_{1}$ with $p$, we get the solution of Eq. (1) as follows:

$$
u(x, y, z, t)=\frac{c p^{2}\left[b_{1}^{2}+4 b_{1} \cos (p(x+c z-\alpha t)) \cos (\Omega y)\right]}{2\left[b_{1} \cos (p(x+c z-\alpha t))+\cos (\Omega y)\right]^{2}}
$$

where

$$
\left\{\begin{array}{l}
u_{0}<\frac{1}{6}\left(c p^{2}-4 \alpha\right), \\
3 \Omega^{2}=c p^{4}+\left(4 \alpha+6 u_{0}\right) p^{2} \\
b_{1}^{2}=\frac{4 \Omega^{2} b_{2}}{\Omega^{2}+c p^{4}} .
\end{array}\right.
$$

The solution represented by Eq. (18) is a doubly periodic solution. This result shows the breather soliton represented by Eq. (15) degenerated into doubly periodic as $u_{0}$ passes through $\frac{1}{6}\left(c p^{2}-4 \alpha\right)$ from the right side to the left side. This is also a bifurcation phenomenon of breather soliton with parameter $u_{0}$ at the special value $\frac{1}{6}\left(c p^{2}-4 \alpha\right)$. This is a new dynamical feature of cross soliton.

By verifying that all the functions represented by Eq. (10), Eq. (12), Eq. (15), and Eq. (18) are the solutions of the YTSF equation under the constraint Eq. (9), Eq. (13), Eq. (16), and Eq. (19), respectively, it is important that the existence of cross-soliton solution Eq. (10), periodic solitary solution Eq. (10), breather soliton Eq. (15), and doubly periodic solution Eq. (18) to YTSF equation is dependant on the different ranges of $u_{0}$, respectively. If we put one and the same velocity $\alpha, p$, then the structure of solution is different in a small neighborhood of $u_{0}=\frac{1}{6}\left(c p^{2}-4 \alpha\right)$ and $u_{0}=-\frac{1}{6}\left(c p^{2}+4 \alpha\right)$, respectively. Cross soliton Eq. (10) changes to periodic solitary solution Eq. (12) when the parameter $u_{0}$ varies from the left side of $u_{0}=-\frac{1}{6}\left(c p^{2}+4 \alpha\right)$ to the right side, which shows soliton degeneracies of cross soliton of the YTSF equation. And when $u_{0}>\operatorname{Max}\left\{\frac{1}{6}\left(c p^{2}-4 \alpha\right), \frac{1}{6}\left(c p^{2}+4 \alpha\right)\right\}$, there occurs coexistence of two kinds of periodic and breather soliton Eq. (12) and Eq. (15). Similarly, the structure of solution is also different in an arbitrary small neighborhood of $u_{0}=\frac{1}{6}\left(c p^{2}-4 \alpha\right)$, doubly periodic solution Eq. (18) changes to breather soliton Eq. (15) as $u_{0}$ varies from the left side of $u_{0}=\frac{1}{6}\left(c p^{2}-4 \alpha\right)$ to the right side. The above results show that the higher dimensional nonlinear evolution equation YTSF has rich dynamics of cross soliton. 


\section{Concluding}

In this paper, we employ the parameter perturbation method for seeking dynamical feature of a general nonlinear partial differential equation. According to the above discussion, we draw the conclusion that for constant equilibrium solution (as a parameter) $u_{0}$ of the YTSF equation there exist two bifurcation values: one is $\frac{1}{6}\left(c p^{2}-4 \alpha\right)$ and another is $-\frac{1}{6}\left(c p^{2}+4 \alpha\right)$, which is the value of soliton degeneracy of cross soliton and retroflexion of breather soliton (light periodic breather changes into dark periodic breather), respectively. Around the both sides at $u_{0}$, the dynamics of solutions is all changed. The dynamics of cross soliton of the YTSF equation is dependent on the value range of equilibrium solution $u_{0}$ in the equilibrium solution space of the YTSF equation. This is a new dynamical feature in a nonlinear spatiotemporal dynamical system. In the future, we intend to study other kinds of dynamics for the YTSF equation.

\section{Acknowledgements}

This study was funded by the National Natural Science Foundation of China(Grants No. 11501474), Fundamental Research Funds for the Central Universities (Grants No. A0920502051813-113), and Sichuan Educational Science Foundation (Grant No. 12ZB069 and No. 13ZB0125).

\section{Funding}

Not applicable.

\section{Competing interests}

The authors declare that there is no conflict of interests regarding the publication of the paper.

\section{Authors' contributions}

The authors declare that this study was accomplished in collaboration with the same responsibility. All authors read and approved the final manuscript.

\section{Publisher's Note}

Springer Nature remains neutral with regard to jurisdictional claims in published maps and institutional affiliations.

Received: 2 December 2018 Accepted: 28 May 2019 Published online: 06 June 2019

\section{References}

1. Yomba, E.: Construction of new soliton-like solutions for the $(2+1)$ dimensional Kadomtsev-Petviashvili equation. Chaos Solitons Fractals 21, 75-79 (2004)

2. Ablowitz, M.J., Clarkson, P.A.: Solitons, Nonlinear Evolution and Inverse Scattering. Cambridge University Press, Cambridge (1991)

3. Zou, D.W., Gao, Y.T., Meng, G.Q., Shen, Y.J., Yu, X.: Multi-soliton solutions of a variable-coefficient KdV equation. Nonlinear Dyn. 75(4), 701-708 (2014)

4. Huang, C.D., Li, H., Cao, J: A novel strategy of bifurcation control for a delayed fractional predator-prey model. Appl. Math. Comput. 347, 808-838 (2019)

5. Huang, C.D., Zhao, X., Wang, X.H., Wang, Z.X., Xiao, M., Cao, J.: Disparate delays-induced bifurcations in a fractional-order neural network. J. Franklin Inst. 356(5), 2825-2846 (2019)

6. Huang, C.D., Cao, J.: Impact of leakage delay on bifurcation in high-order fractional BAM neural networks. Neural Netw. 98, 223-235 (2018)

7. Huang, C.D., Song, X.Y., Fang, B.: Modeling, analysis and bifurcation control of a delayed fractional-order predator-prey model. Int. J. Bifurc. Chaos 28(9), 1850117 (2018)

8. Dai, Z.D., Wang, C.J., Liu, J.: Inclined periodic homoclinic breather and rogue waves for the (1 + 1)-dimensional Boussinesq equation. Pramana J. Phys. 83(4), 473-480 (2014)

9. Xu, Z.H., Chen, H.L., Jiang, M.R., Dai, Z.D., Chen, W.: Resonance and deflection of multi-soliton to the (2 + 1)-dimensional Kadomtsev-Petviashvili equation. Nonlinear Dyn. 78(1), 461-466 (2014)

10. Chen, W., Chen, H.L., Dai, Z.D.: Rational homoclinic solution and rogue wave solution for the coupled long-wave-short-wave system. Pramana J. Phys. 86(3), 713-717 (2016)

11. Ablowitz, M.J., Clarkson, P.A.: Soliton, Nonlinear Evolution Equations and Inverse Scattering. Cambridge University Press, New York (1991)

12. Gu, C.H.: Soliton Theory and Its Application. Zhejiang Science and Technology Press, Zhejiang (1990)

13. Wadati, M., Sanuki, H., Konno, K.: Relationships among inverse method, Bäcklund transformation and an infinite number of conservation laws. J. Phys. Soc. Jpn. 53(2), 419-436 (1975)

14. Yajima, T., Wadati, M.: Soliton solution and its property of unstable nonlinear Schrodinger equation. J. Phys. Soc. Jpn. $59,41-47(1990)$

15. Hirota, R.: Exact solution of the Korteweg-de Vries equation for multiple collisions of solitons. Phys. Rev. Lett. 27(18), 1192-1194 (1971) 
16. Lou, S.Y., Huang, G.X., Ruan, H.Y.: Exact solitary waves in a convecting fluid. J. Phys. A, Math. Gen. 24, L584-L590 (1991)

17. Parkes, E.J., Duffy, B.R.: An automated tanh-function method for finding solitary wave solutions to non-linear evolution equations. Comput. Phys. Commun. 98(3), 288-300 (1996)

18. Li, B., Chen, Y.: On exact solutions of the nonlinear Schrodinger equations in optical fiber. Chaos Solitons Fractals 21(1), 241-247 (2004)

19. Yu, S.J., Toda, K., Sasa, N., Fukuyama, T.: N soliton solutions to the Bogoyavlenskii-Schiff equation and a quest for the soliton solution in (3+1) dimensions. J. Phys. A, Math. Gen. 31, 3337-3347 (1998)

20. Schff, J.: Painleve Transendent, Their Asymptotics and Physical Applications. Plenum, New York (1992)

21. Dai, Z.D., Liu, J., Li, D.L.: Applications of HTA and EHTA to YTSF equation. Appl. Math. Comput. 207, 360-364 (2009)

22. Roshid, H.O.: Lump solutions to a (3 + 1)-dimensional potential-Yu-Toda-Sasa-Fukuyama (YTSF) like equation. Int. J. Appl. Comput. Math. 3, 1455-1461 (2017)

23. Yan, Z.Y.: New families of nontravelling wave solutions to a new $(3+1)$-dimensional potential-YTSF equation. Phys. Lett. A 318, 78-83 (2003)

24. Gardner, C.S., Greene, J.M., Kruskal, M.D., Miura, R.M.: Method for solving the Korteweg-de Vries equation. Phys. Rev. Lett. 19, 1095-1097 (1967)

25. Woopyo, H., Jung, Y.D.: Auto-Backlund transformation and analytic solutions for general variable-coefficient KdV equation. Phys. Lett. A 257, 149-152 (1999)

26. Dai, Z.D., Jiang, M.R., Dai, Q.Y., Li, S.L.: Homoclinic bifurcation for Boussinesq equation with even constraint. Chin. Phys. Lett. 23(5), 1065-1067 (2006)

27. Hu, Y.J., Chen, H.L., Dai, Z.D.: New kink multi-soliton solutions for the (3+1)-dimensional potential Yu-Toda-Sasa-Fukuyama equation. Appl. Math. Comput. 234, 548-556 (2014)

28. Yin, H.M., Tian, B., Cha, J.X., Wu, Y., Sun, W.R.: Solitons and bilinear Backlund transformations for a $(3+1)$-dimensional Yu-Toda-Sasa-Fukuyama equation in a liquid or lattice. Appl. Math. Lett. 58, 178-183 (2016)

\section{Submit your manuscript to a SpringerOpen ${ }^{\circ}$ journal and benefit from:}

- Convenient online submission

- Rigorous peer review

- Open access: articles freely available online

- High visibility within the field

Retaining the copyright to your article

Submit your next manuscript at $>$ springeropen.com 